\title{
Cahiers « Mondes anciens »
}

\section{Le rôle des pontifes dans l'expiation des prodiges à Rome, sous la République : le cas des "procurations" anonymes}

Yann Berthelet

\section{(2) OpenEdition}

Journals

Édition électronique

URL : http://journals.openedition.org/mondesanciens/348

DOI : $10.4000 /$ mondesanciens.348

ISSN : 2107-0199

Éditeur

UMR 8210 Anthropologie et Histoire des Mondes Antiques

Référence électronique

Yann Berthelet, "Le rôle des pontifes dans l'expiation des prodiges à Rome, sous la République : le cas des "procurations" anonymes », Cahiers « Mondes anciens » [En ligne], 2 | 2011, mis en ligne le 20 juillet 2011, consulté le 24 octobre 2019. URL : http://journals.openedition.org/mondesanciens/348 ; DOI : 10.4000/mondesanciens.348

Ce document a été généré automatiquement le 24 octobre 2019.

\section{(c) (i) (9)}

Les Cahiers «Mondes Anciens » sont mis à disposition selon les termes de la licence Creative Commons Attribution - Pas d'Utilisation Commerciale - Pas de Modification 4.0 International. 


\title{
Le rôle des pontifes dans l'expiation des prodiges à Rome, sous la République : le cas des "procurations" anonymes
}

\author{
Yann Berthelet
}

1 On peut définir le prodige ${ }^{1}$, à Rome, comme un signe divin oblatif, distinct du présage en ce sens qu'il n'annonce l'avenir - un avenir lourd de menaces - que dans l'hypothèse où les humains négligeraient de prendre les mesures d'expiation nécessaires pour apaiser les dieux ${ }^{2}$. Il consiste en un phénomène ou un comportement inquiétant par son anormalité, son étrangeté, son caractère extraordinaire - mais pas nécessairement contre nature (BOUCHÉ-LECLERCQ 1882, p. 75 sq.) - ; il est observable à tout moment, contrairement à l'auspicium oblatif, observable uniquement dans le cadre délimité d'une action précise (LINDERSKI 1995, p. 613 sq.) ; il déclenche dans la collectivité une angoisse suffisamment importante pour être interprété comme un signe négatif signifiant la colère des dieux (ira deorum) et la rupture de la pax deorum, à savoir les «rapports réguliers entre le monde humain et le monde surnaturel» (BLOCH 1949, p. 119). Si cette définition ne saurait épuiser toutes les nuances de sens des termes prodigium, portentum, ostentum ou monstrum ${ }^{3}$, elle rend cependant assez bien compte de la manière dont les prêtres, les magistrats et les sénateurs romains interprétaient généralement les prodiges publics. Une fois que les autorités avaient reconnu que les prodiges rapportés concernaient le peuple romain, elles engageaient un processus de conjuration et d'expiation de ces prodiges (procuratio) afin d'apaiser les divinités irritées et de rétablir la pax deorum. Le Sénat consultait alors les prêtres sur les mesures d'expiation nécessaires (piacula). Trois types de prêtres étaient compétents, à Rome, en matière de prodiges : les pontifes romains ; les (quin) décemvirs sacris faciundis romains, chargés de la consultation des Livres oraculaires de la Sibylle ; les haruspices étrusques.

2 Les pontifes se seraient vu confier par Numa la prise en charge des prodiges ${ }^{4}$. L'examen des sources relatives aux périodes royale et républicaine nous amène cependant à 
constater que leur intervention dans le processus d'expiation des prodiges ne nous est signalée explicitement qu'à 15 reprises, contre 53 pour les (quin) décemvirs et 36 pour les haruspices, pourtant étrangers 5 . Face à ce paradoxe, il peut être intéressant de se demander, à la suite de Jacqueline CHAMPEAUX (1996), s'il ne faudrait pas attribuer aux pontifes les nombreuses procurations anonymes, appelées unspecified dans la bibliographie anglo-saxonne, à savoir celles où l'institution qui assure la prise en charge du prodige n'est pas précisée. Par «attribution aux pontifes », nous entendons que les pontifes auraient été consultés sur les mesures d'expiation à prescrire dans le sénatus-consulte : le Sénat n'aurait donc pas décidé de lui-même des piacula nécessaires pour apaiser les dieux. Voici deux exemples-types de ces expiations anonymes : horum prodigiorum causa diem unum supplicatio fuit ${ }^{6}$; ea prodigia hostiis maioribus procurata ${ }^{7}$.

Derrière cet anonymat, plutôt que les pontifes, on a généralement pensé que se cachait le seul Sénat, ce qui impliquerait que l'assemblée puisse décider de procurations de prodiges sans en référer aux prêtres. Ainsi Ludwig wÜLKER (1903, p. 29 sq.) affirme, sans autre preuve que le silence de Tite-Live, que, dans les cas simples et sans surprise, le sénat ordonne de son propre chef les mesures à prendre, sans consulter au préalable les autorités sacerdotales. Cette thèse a souvent été reprise par la suite, en particulier par Raymond Bloch et Michel Hano. Pour Raymond Bloch, « lors des prodiges bien connus, fréquents et de moyenne importance, le Sénat peut ordonner immédiatement les cérémonies qui lui paraissent s'imposer et il confie l'exécution aux consuls ou bien aux pontifes. Parfois, lors de prodiges graves, cette même décision est prise, mais s'accompagne d'un recours aux spécialistes des procurations. Le plus souvent l'affaire est renvoyée devant ceux-ci et aucune cérémonie n'est décidée sans leur avis » (BLOCH 1963, p. 121 sq.). Pour Michel Hano, "parfois, le Sénat décide seul de la conduite à suivre et de fait il semble pour les prodiges les plus courants savoir quelle attitude il convient d'adopter. En effet, comme l'a noté Bouché-Leclercq, «les Pontifes romains avaient aussi dans leurs Annales un recueil qui s'accroissait de toutes les consultations soit révélées soit demandées aux livres sibyllins et aux haruspices, consultations valables pour des cas identiques ", et de telles connaissances devaient être partagées par le Sénat et par les consuls. Ainsi la procuratio pour la pluie de pierre rappelée en 1, 31,1 qui consiste en une neuvaine servira de référence quand le même phénomène se reproduira: mansit certe sollemne ut, quandoque idem prodigium nuntiaretur, feriae per novem dies agerentur ${ }^{8}$. Souvent donc en face d'un grand nombre de prodiges, le Sénat décide seul » (HANO 1986, p. 105).

4 Cette hypothèse selon laquelle l'anonymat des expiations unspecified cache un Sénat apte à décider seul de certaines procurations, sans recours systématique au consilium sacerdotal, rencontre à mon avis au moins trois objections, qui amènent plutôt à attribuer ces procurations anonymes aux pontifes.

5 La première objection est que cette interprétation des textes va absolument à l'encontre de la logique des institutions romaines, telle qu'elle a été dégagée par les études les plus récentes. Les travaux de John Scheid, par exemple, ont ainsi montré qu'en matière de prodiges l'avis des prêtres, en particulier des pontifes, s'il n'est certes que consultatif, doit cependant être sollicité par les magistrats et le Sénat: "Nous savons, explique-t-il, que les consuls et le sénat n'agissaient pas seuls lors des séances inaugurales de l'année. Les sources nous informent que l'exécution de piacula suscités par les prodiges annoncés se faisait ex decreto pontificum. Nous savons également que les 
pontifes étaient consultés à propos des problèmes relatifs aux vœux » (sCHEID 1994, p. 154).

6 La deuxième objection réside paradoxalement dans la rareté de la mention explicite des expiations pontificales. Ce constat, loin d'aller dans le sens de la thèse d'une identification, derrière les procurations anonymes, du Sénat agissant seul, la rend au contraire intenable. Car une telle position réduirait les interventions des pontifes à un nombre absolument insignifiant, non seulement en chiffre absolu (15), mais aussi par rapport au nombre d'interventions des (quin) décemvirs (53), et même des haruspices (36), pourtant étrangers ${ }^{9}$ ! Il serait en effet surprenant que l'intervention des pontifes, par ailleurs centrale dans tout le reste du domaine religieux à Rome ${ }^{10}$, soit réduite à la portion congrue pour les prodiges, alors même que Numa, d'après la tradition, les en avait chargés : ut idem pontifex edoceret, quaeque prodigia fulminibus aliove quo visu missa susciperentur atque curarentur ${ }^{11}$. Il serait tout aussi incohérent qu'ils interviennent si peu dans le processus d'expiation des prodiges, quand on sait que c'était à eux que revenait le rôle d'enregistrer les prodiges survenus et de rédiger chaque année la chroniquebilan des passifs du peuple romain envers les dieux: "Le souverain pontife, rappelle John Scheid, possédait le pouvoir d'accueillir les annonces de prodiges tout au long de l'année, de les enregistrer après les avoir vérifiées et discutées avec ses collègues (...) Il obtenait ainsi toutes les données indispensables pour décider (...) de l'état des relations avec les dieux. Quant à la tabula affichée, je préfère la considérer comme un décret (ou une suite de décrets) : comme beaucoup d'édits ou de décrets, celui-ci était affiché à la résidence officielle de celui qui l'avait pris. C'est vraisemblablement en fin d'année que le souverain pontife «bouclait » sa chronique, la rédigeait sous forme de décret, remis aux consuls et aux sénateurs à leur requête et publié dans la résidence du souverain pontife » (sCHEID 1994, p. 154 sq.). Si l'on suit cette hypothèse, fort convaincante, faisant de la chronique du souverain pontife un décret sacerdotal, il faut en tirer toutes les conséquences et en déduire - ce qui, à ma connaissance, n'a jamais été fait - une consultation systématique des pontifes en matière de prodiges. Voici la reconstitution que je propose du parcours institutionnel qui résulterait d'une telle hypothèse : les consuls et le Sénat demandaient en début d'année au collège pontifical sa "chronique-décret " des événements religieux, prodiges y compris, de l'année écoulée, ce qui impliquait à la fois l'énoncé des prodiges enregistrés et la recommandation de mesures d'expiation. Lorsque les pontifes le jugeaient nécessaire, ils recommandaient en outre, dans cette même "chronique-décret", de compléter les conjurations immédiates qu'ils conseillaient par des mesures d'expiation complémentaires, sur avis des (quin) décemvirs sacris faciundis, après consultation des Livres sibyllins, ou sur avis des haruspices étrusques.

7 Si l'on a eu du mal à repérer cette consultation systématique et préalable des pontifes et les mesures d'expiation immédiates qu'ils recommandaient dans leur « chroniquedécret ", c'est non seulement parce que les sources éludent généralement cette étape du processus de conjuration pour se concentrer, lorsqu'elle avait lieu, sur l'intervention finale des (quin) décemvirs ou des haruspices, laquelle était plus exceptionnelle et avait donc un fort intérêt dramatique; mais aussi, dans les rares cas où cette première vague d'expiations est signalée, parce que les sources font disparaître le décret pontifical derrière le sénatus-consulte qui le rendait exécutoire ${ }^{12}$. Voici trois passages de Tite-Live qui échappent en partie à la brevitas habituelle des sources :

Decretum ut ea prodigia, partim maioribus hostiis, partim lactentibus procurarentur, et uti supplicatio per triduum ad omnia pulvinaria haberetur; cetera, cum decemviri libros 
inspexissent, ut ita fierent, quem ad modum cordi esse divis $<e>$ carminibus praefarentur, "Le Sénat décida de conjurer ces prodiges par le sacrifice ou de victimes adultes ou d'animaux à la mamelle et de procéder à des prières publiques pendant trois jours auprès de tous les lits de parade; une fois que le collège des décemvirs aurait consulté les Livres, on satisferait les dieux suivant les prescriptions en vers " (TiteLive, 22, 1, 15-16).

His prodigiis cladibusque anxii patres decreverunt ut et consules quibus diis videretur hostiis maioribus sacrificarent et decemviri libros adirent, "Ces prodiges et ce fléau inquiétaient les pères qui prirent la décision suivante : les consuls offriraient des victimes adultes aux dieux de leur choix et les décemvirs consulteraient les Livres » (Tite-Live, 40, 19, 4).

Priusquam consules in provincias proficiscerentur, prodigia per pontifices procurari placuit... Ea procurata, Latinaeque instauratae, quod Laurentibus < pars > carnis quae dari debet data non fuerat. Supplicatio quoque earum religionum causa fuit quibus diis decemviri ex libris ut fieret ediderunt, «Le sénat invita les consuls à conjurer les prodiges sous l'autorité des pontifes avant de rejoindre leur poste... Les prodiges furent conjurés. Aux Féries Latines, les Laurentes avaient été privés de la part de viande à laquelle ils avaient toujours eu droit : on recommença les cérémonies. Pour rétablir la paix des consciences, les décemvirs, après consultation des Livres sibyllins, désignèrent les dieux auxquels on devait s'adresser » (Tite-Live, 37, 3, 1 et 4-5, cas compliqué par l'épisode des Féries Latines).

8 La troisième objection à l'attribution au Sénat seul des conjurations anonymes résulte des recoupements que l'on peut faire entre les listes de prodiges dont la procuration est anonyme et les listes de prodiges expiés par les pontifes. Le tableau proposé ci-après tente de mettre en évidence, à travers quelques exemples, l'intérêt de cet argument comparatif, qui n'a encore jamais été développé : 


\section{Tite-Live, 37, 3, 1-4 \\ Priusquam consules in provincias proficiscerentur, prodigia per pontifices procurari placuit. Romae Iunonis Lucinae templum de caelo tactum erat ita ut fastigium valvaeque deformarentur; Puteolis pluribus locis murus et porta fulmine icta et duo homines exanimati; Nursiae sereno satis constabat nimbum ortum, ibi quoque duos liberos homines exanimatos; terra apud se pluvisse Tusculani nuntiabant, et Reatini mulam in agro suo peperisse. Ea procurata..., "Le sénat invita les consuls à conjurer les prodiges sous l'autorité des pontifes avant de rejoindre leur poste. À Rome, la foudre était tombée sur le temple de Junon Lucine, endommageant le toit et la porte. À Pouzzoles, les remparts avaient été touchés par la foudre en plusieurs endroits ainsi qu'une porte, deux personnes avaient trouvé la mort. À Nursie, alors que le ciel était pur, on avait vu un orage se former et on déplora, ici encore, la mort de deux hommes libres. Les Tusculans signalèrent qu'il $\mathrm{y}$ avait eu des pluies de terre chez eux; à Réate une mule avait mis bas. Les prodiges furent conjurés ».}

Tite-Live, 25, 7, 7-9

Tempestates foedae fuere; in Albano monte biduum continenter lapidibus pluvit; tacta de caelo multa, duae in Capitolio aedes, vallum in castris multis locis supra Suessulam, et duo vigiles exanimati; murus turresque quaedam Cumis non ictae modo fulminibus sed etiam decussae. Reate saxum ingens visum volitare, sol rubere solito magis sanguineoque similis. Horum prodigiorum causa diem unum supplicatio fuit et per aliquot dies consules rebus divinis operam dederunt et per eosdem dies sacrum novendiale fuit, "On eut d'affreuses tempêtes, des pluies de pierre s'abattirent sans arrêt sur le mont Albain pendant deux jours; la foudre frappa souvent: elle tomba deux fois sur les temples du Capitole, à plusieurs reprises sur le retranchement $d u$ camp au-dessus de Suessula, faisant deux morts. Les fortifications de Cumes et plusieurs tours reçurent aussi la foudre et furent même détruites. À Réate, on vit voler une énorme pierre et le soleil parut d'un rouge inaccoutumé, couleur de sang. Il y eut une journée de prières publiques pour conjurer ces prodiges; les consuls s'occupèrent des cérémonies religieuses pendant un certain nombre de jours qui furent aussi marqués par une neuvaine ».

Tite-Live, 26, 23, 4-6

Eodem anno prodigia aliquot visa nuntiataque sunt. In aede Concordiae Victoria quae in culmine erat fulmine icta decussaque (...) et Anagniae et Fregellis nuntiatum est murum portasque de caelo tacta, et in foro Subertano sanguinis rivos per diem totum fluxisse, et Ereti lapidibus pluvisse, et Reate mulam peperisse. Ea prodigia hostiis maioribus sunt procurata et obsecratio in unum diem populo indicta et novendiale sacrum, « La même année des prodiges furent constatés et signalés : la Victoire qui se trouvait en haut du temple de la Concorde fut frappée de la foudre et abattue (...). À Anagni et à Frégelles on signala que les remparts et les portes avaient reçu la foudre; sur le forum de Subertum du sang avait coulé pendant toute une journée. À É rétum on avait eu une pluie de pierres et à Réate une mule avait mis bas. On sacrifia des victimes adultes pour conjurer ces prodiges et on ordonna une journée de prières publiques suivie d'un sacrifice huit jours plus tard ». 
Pour plus de visibilité, j'ai mis à chaque fois en caractères gras, dans le tableau, les prodiges similaires entre la liste de prodiges à conjuration pontificale $(37,3,1-4)$ et les deux listes à conjuration anonyme $(25,7,7-9$ et $26,23,4-6)$. Dans les deux types de listes, nous trouvons des murs et des portes frappés par la foudre (Puteolis pluribus locis murus et porta fulmine icta en 37, 3, 2/Anagniae et Fregellis nuntiatum est murum portasque de caelo tacta en $26,23,5$ ), des dégâts causés à des temples par la foudre (Romae Iunonis Lucinae templum de caelo tactum erat ita ut fastigium valvaeque deformarentur en 37, 3, 2/In aede Concordiae Victoria quae in culmine erat fulmine icta decussaque en 26, 23, 4 et tacta de caelo multa, duae in Capitolio aedes en 25, 7, 7), deux personnes trouvant la mort (duo homines exanimati en 37, 3, 2 et ibi quoque duos liberos homines exanimatos en 37, 3, 3/duo vigiles exanimati en $25,7,7$ ), et deux mules mettant bas à Réate (Reatini mulam in agro suo peperisse en 37, 3, 3/Reate mulam peperisse en $26,23,5$ ). Ces grandes similitudes nous autorisent à supposer que c'est bien une intervention pontificale qui se cache derrière les conjurations anonymes : cette remarque vaut surtout pour Tite-Live, car les autres sources s'intéressent trop irrégulièrement aux prêtres sollicités en cas de prodiges pour qu'on puisse se risquer, à leur égard, à formuler la même hypothèse. Cette intervention pontificale était suffisamment habituelle et connue de tous pour que les auteurs anciens ne voient pas la nécessité de la rappeler systématiquement à leurs lecteurs ni l'intérêt dramatique de la signaler dans leur récit, contrairement à la consultation des Livres Sibyllins par les (quin) décemvirs ou à l'intervention des haruspices étrusques, plus exceptionnelles.

À ces rapprochements entre prodiges de nature similaire, on pourrait objecter les remarques d'Annie Vigourt sur l'impossibilité d'énumérer les types de présages, signes très proches des prodiges ${ }^{13}$ : "l'émotion produite étant ce qui distinguait le signe omineux d'un phénomène sans signification particulière, tout essai pour énumérer les types de signes acceptés comme véritables est voué à l'échec : tout peut être signe, il n'y a pas de spécialisation ou de catégories "signifiant » automatiquement "quelque chose » de précis » (VIGOURT 2001, p. 93). L'examen des sources confirme en effet que l'émotion ressentie face à un objet (phénomène ou comportement) était la plupart du temps bien plus déterminante que la nature même de l'objet, dans la reconnaissance de celui-ci comme présage ou prodige. Il ne faudrait pas pour autant inverser de manière trop rigide le raisonnement : avant que l'émotion ne construise l'objet comme prodige, elle est elle-même suscitée, bien souvent, par une certaine étrangeté propre à l'objet. Annie Vigourt ne dit pas autre chose lorsqu'elle affirme que «le présage est quelque chose d'étrange » (VIGOURT 2001, p. 94). C'est encore plus frappant avec les prodiges. Il arrivait certes régulièrement, en raison d'un contexte particulièrement angoissant, que soient interprétés comme prodiges des phénomènes qui n'avaient rien d'étrange - ce que les sources ne manquent pas, alors, de souligner ${ }^{14}-$, comme dans les cas de clades ou de nefas. Il serait toutefois erroné de penser que l'étrangeté ne peut pas être intrinsèque à l'objet, qu'elle est nécessairement induite du contexte : un animal ou un humain monstrueux, un tremblement de terre, une éclipse ou l'irruption en ville d'un animal sauvage, sont en effet perçus par les Romains comme étranges en soi. Et c'est leur étrangeté qui se communique ensuite au contexte et lui donne sens : « [l'étrangeté des signes omineux] s'intègre dans un ensemble qui devient compréhensible grâce à elle » (VIGOURT 2001, p. 94). Tous ces phénomènes et comportements étranges ne sont pas pour autant ipso facto des présages ou des prodiges; ils ne le sont que potentiellement, en raison de leur étrangeté intrinsèque. Un contexte angoissant ou un 
caractère de nouveauté, de répétition ou de durée particulièrement inquiétante reste nécessaire pour susciter l'émotion qui permettra aux témoins du phénomène ou du comportement d'en remarquer l'étrangeté et de le reconnaître comme signe. Il paraît d'autant plus légitime de s'interroger sur les types de phénomènes ou de comportements potentiellement interprétables comme prodiges, que l'on ne parle ici que des prodiges reconnus comme prodigia publica, c'est-à-dire de ceux qui ont été retenus, enregistrés et expiés par les autorités romaines. La distanciation impliquée par le processus d'enregistrement et de sélection des prodiges, et par les discussions entre pontifes et entre sénateurs auxquelles ce processus institutionnel donnait lieu, relativisait nécessairement la composante émotionnelle du prodige, au profit de la nature du prodige annoncé. L'émotion n'était évidemment pas entièrement évacuée, non seulement parce que prêtres, sénateurs et magistrats n'étaient pas à l'abri de l'émotion suscitée par les prodiges, mais surtout parce qu'il leur fallait tenir compte de l'émotion produite pour mieux la canaliser et la résorber. Mais les listes pontificales de prodiges qui nous sont indirectement parvenues, via les annalistes, nous présentent un regard technique et détaché, la plupart du temps, de toute émotion : à l'énumération assez sèche des prodiges, succède l'énoncé des mesures d'expiation. Une prise en compte quasiment automatique de certains types de prodiges est même parfois discernable, notamment pour les pluies de pierres ${ }^{15}$.

11 Une seconde objection à ce recoupement de listes pourrait être formulée : les (quin) décemvirs et les haruspices expient souvent, entre autres, nombre de prodiges présents dans les listes à conjuration explicitement pontificale. En Tite-Live 35, 9, 2-5, par exemple, les décemvirs conjurent des prodiges de foudre tout à fait banals, du même type que ceux que nous avons rencontrés ci-dessus, en Tite-Live 37, 3, 2 :

Aquae ingentes eo anno fuerunt, et Tiberis loca plana Urbis inundavit. Circa portam Flumentanam etiam conlapsa quaedam ruinis sunt, et porta Caelimontana fulmine icta est, murusque circa multis locis de caelo tactus, et Ariciae et Lanuvii et < in > Aventino lapidibus pluit, et a Capua nuntiatum est examen vesparum ingens in forum advolasse et in Martis aede consedisse; eas conlectas cum cura et igni crematas esse. Horum prodigiorum causa decemviri libros adire iussi, et novemdiale sacrum factum, et supplicatio indicta est atque urbs lustrata, «Le Tibre connut de fortes crues cette année-là et le bas de la ville fut inondé; des maisons s'effondrèrent près de la porte Flumentane. La porte $\mathrm{du}$ mont Caelius reçut la foudre ainsi que le mur attenant et beaucoup d'autres endroits. À Aricie, à Lanuvium et sur l'Aventin il y eut des pluies de pierres. On signala qu'à Capoue un énorme essaim d'abeilles s'était posé au forum et installé dans le temple de Mars: on le recueillit avec soin et on le brûla. Les décemvirs furent invités à consulter les Livres à cause de ces prodiges; on fit une neuvaine, des prières publiques furent prescrites et [on procéda à la lustration de] la ville $»^{16}$.

Mais cette objection tombe si l'on prend en compte les arguments développés plus haut en faveur du caractère systématique et préalable de la consultation pontificale, suivie de la publication de leur "chronique-décret» religieuse annuelle. Les passages semblables à l'extrait ci-dessus cité nous abusent vraisemblablement par leur brevitas, que j'ai mise en évidence plus haut à l'aide de trois passages de Tite-Live qui y échappent et laissent apparaître une vague de conjurations antérieure à l'intervention décemvirale ${ }^{17}$. En Tite-Live 35, 9, 2-5, les décemvirs conjurent bien, lors d'une vague d'expiations complémentaires, l'ensemble des prodiges de la liste, y compris les prodiges de foudre - la structure de la liste, avec horum prodigiorum causa, ne laissant guère de doute sur la conjuration en bloc des prodiges -, mais après une première conjuration par les pontifes et non à leur place. 
13 Le rôle majeur des pontifes dans les institutions religieuses de Rome, en particulier le souvenir que conserve la tradition du rôle que Numa leur attribua en matière de prodiges; l'hypothèse de John Scheid d'une chronique pontificale rédigée sous forme de décret $\mathrm{du}$ collège sacerdotal; l'hypothèse que j'en tire d'une consultation systématique des pontifes, préalable à l'éventuelle consultation des (quin) décemvirs et des haruspices pour des mesures d'expiation complémentaires; et l'existence d'une grande similitude entre les prodiges des listes à conjuration anonyme et ceux des listes à conjuration pontificale, constituent un faisceau d'indices allant dans le sens de l'intuition de Jacqueline Champeaux : «peut-on, pour autant, conclure, disait-elle, que, dans les expiations "unspecified", les intervenants, non précisés, sont les pontifes? L'hypothèse est séduisante, même si aucune preuve solide, je veux dire positive, ne permet de l'étayer » (CHAMPEAUX 1996, p. 71 sq., n. 17).

\section{Annexe}

\begin{tabular}{|c|c|}
\hline $\begin{array}{l}\text { Date de } \\
\text { l'intervention } \\
\text { sacerdotale }^{18} \text {. }\end{array}$ & $\begin{array}{l}\text { Principales sources permettant } d^{\prime} \text { 'identifier l'intervention sacerdotale en } \\
\text { lien avec les prodiges survenus }{ }^{19} \text {. }\end{array}$ \\
\hline \multicolumn{2}{|c|}{ Interventions des pontifes (15 explicites, 2 incertaines) } \\
\hline 483 & 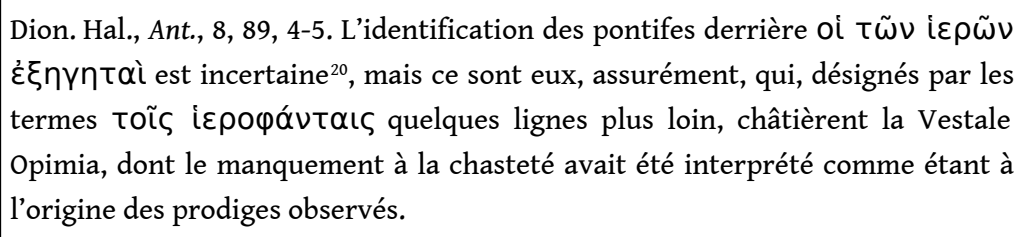 \\
\hline 364 & Oros., 3, 4, 1-6; Aug., Civ., 2, 8 \\
\hline 228 & Oros., $4,13,3$ \\
\hline 217 & Liv., 22, 9, 7-11 \\
\hline 216 & Liv., 22, 57, 2-6 \\
\hline 213 & Liv., 24, 44, 7-9 \\
\hline 210 & Liv., 27, 4, 11-15 \\
\hline 207 & Liv., 27, 37, 1-10 \\
\hline 203 & Liv., 30, 2, 9-13 \\
\hline 194 & Liv., $34,45,6-8$ \\
\hline 190 & Liv., 37, 3, 1-6 \\
\hline 186 & Liv., $39,22,1-5$ \\
\hline 180 & Liv., $40,36,14$ et $40,37,1-3$ \\
\hline
\end{tabular}




\begin{tabular}{|c|c|}
\hline 176 & Liv., $41,16,6$ \\
\hline 37 & Serv., Georg., 2, 162 \\
\hline 472 & 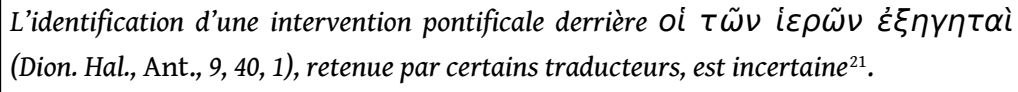 \\
\hline 206 & $\begin{array}{l}\text { L'extinction du feu de Vesta, expiée par des sacrifices et une supplicatio (Liv., 28, 11, } \\
\text { 6-7), ne semble pas avoir été interprétée comme un prodige, mais comme une simple } \\
\text { négligence humaine, pour laquelle des piacula étaient néanmoins nécessaires. }\end{array}$ \\
\hline \multicolumn{2}{|c|}{ Interventions des (quin) décemvirs sacris faciundis (53 explicites, 4 incertaines) } \\
\hline $\begin{array}{l}\text { Sous Tarquin le } \\
\text { Superbe }\end{array}$ & Serv., Aen., 2, 140 \\
\hline 504 & Plut., Publ., 21, 2-3 \\
\hline 496 & Dion. Hal., Ant., 6, 17, 2-4 \\
\hline 461 & Liv., 3, 10, 5-7 ; Dion. Hal., Ant., 10, 2, 2-6 \\
\hline 436 & Liv., $4,21,5$ \\
\hline 433 & Liv., 4, 25, 3 \\
\hline 399 & Liv., 5, 13, 4-8 ; Dion. Hal., Ant., 12, frg. 9 \\
\hline 362 & 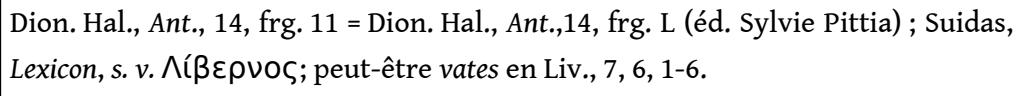 \\
\hline 348 & Liv., 7, 27, 1 \\
\hline 344 & Liv., 7, 28, 6-8 \\
\hline 295 & Liv., $10,31,8$ \\
\hline 293 & Liv., $10,47,6$; Oros., $3,22,5$ \\
\hline 266 & Oros., 4, 5, 7 ; Aug., Civ., 3, 17 \\
\hline 249 & Cens., 17, 8 ; Sch. Hor., Carm. saec., 8 \\
\hline 228 & $\begin{array}{l}\text { Cass. Dio, 12, frg. 50. Cf. Plut., Marcell., 3, 6-7 ; Cass. Dio, } 12 \text { in Zon., 8, } 19 \text { et } \\
\text { Tzetz., ad Lykophr. Alex., } 603 \text {; Oros., 4, 13, 3. }\end{array}$ \\
\hline 218 & Liv., 21, 62 \\
\hline $217(a)$ & Liv., 22, 1, 8-19 et peut-être Macr., Sat., 1, 6, 13-14 \\
\hline $217(b)$ & Liv., 22, 9, 7-11 \\
\hline
\end{tabular}




\begin{tabular}{|c|c|}
\hline $216(a)$ & Liv., 22, 36, 6-8 \\
\hline $216(b)$ & Liv., 22, 57, 2-6. Cf. Plut., Fab., 18, 3 \\
\hline 207 & Liv., 27, 37, 11-15 \\
\hline 205 & Liv., 29, 10, 4-5 ; App., Hann., 56, 233 \\
\hline 200 & Liv., 31, 12, 5-10 \\
\hline $193(a)$ & Liv., 34, 55, 1-4 \\
\hline $193(b)$ & Liv., 35, 9, 3-5 \\
\hline 191 & Liv., 36, 37, 1-6 \\
\hline 190 & Liv., 37, 3, 1-6 \\
\hline 188 & Liv., $38,36,4$ \\
\hline 187 & Liv., 38, 44, 7-8 \\
\hline 183 & Liv., 39, 46, 2-5 \\
\hline 181 & Liv., 40, 19, 1-5 ; Obseq., 6 \\
\hline 180 & Liv., $40,36,14$ et $40,37,1-3$ \\
\hline 179 & Liv., 40, 45, 1-5 \\
\hline 174 & Liv., 41, 21, 10-13 \\
\hline 173 & Liv., 42, 2, 3-7 \\
\hline 172 & Liv., 42, 20, 1-6 \\
\hline 169 & Liv., 43, 13, 7-8 \\
\hline 167 & Liv., $45,16,5-6$ \\
\hline 165 & Obseq., 13 \\
\hline 143 & Obseq. 21 ; Cass. Dio, 22, frg. 74 \\
\hline 142 & obseq., 22 \\
\hline 133 & Cic., Verr., 2, 4, 108 ; Val. Max., 1, 1c ; Lact., Inst., 2, 4, 29 ; Diod., 34/35, frg. 10 \\
\hline 125 & Phleg., Mir., 10 \\
\hline 118 & Obseq., 35 \\
\hline
\end{tabular}




\begin{tabular}{|c|c|}
\hline 117 & Cic., Div., 1, 97-98 \\
\hline 114 & Plut., qu. R., 83 \\
\hline 108 & Obseq., 40 \\
\hline 102 & Obseq., 44 \\
\hline 87 & Granius Licinianus, 35, 1-2 \\
\hline 56 & Cass. Dio, 39, 15-16 \\
\hline 54 & Cass. Dio, de 39, 60, 4 à 39, 61, 4 \\
\hline 50 & Plin., Nat., 17, 243 \\
\hline 38 & Cass. Dio, $48,43,4-6$ \\
\hline $\begin{array}{l}\text { Sous Tarquin le } \\
\text { Superbe }\end{array}$ & 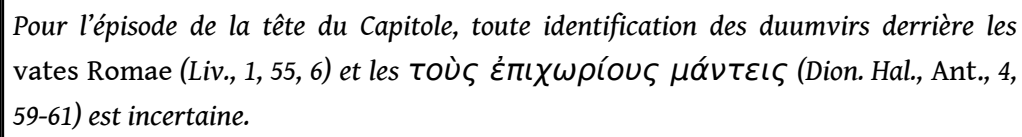 \\
\hline 483 & 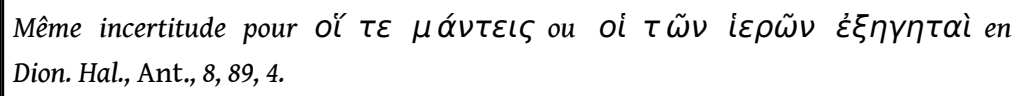 \\
\hline 472 & 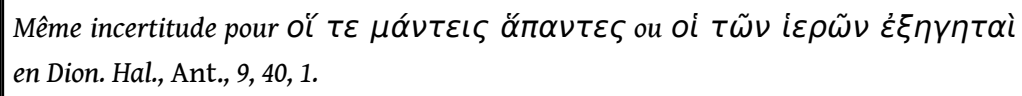 \\
\hline 98 & $\begin{array}{l}\text { En Obseq., } 47, \text { rien ne prouve que les décemvirs soient intervenus dans l'expiation des } \\
\text { prodiges. }\end{array}$ \\
\hline \multicolumn{2}{|c|}{ Interventions des haruspices ${ }^{22}$ ( 36 explicites, 9 incertaines) } \\
\hline À l'époque royale & Plin., Nat., 7, 68-69 \\
\hline $\begin{array}{l}\text { Sous Tarquin le } \\
\text { Superbe }\end{array}$ & $\begin{array}{l}\text { Episode de la tête du Capitole : Liv., 1, 55, 5-6 ; Dion. Hal., Ant., 4, 59-61 ; Plin., } \\
\text { Nat., 28, } 15 \text {; Serv., Aen., 8, 345. Cf. Arnob., 6, } 7\end{array}$ \\
\hline $\mathrm{V}^{\mathrm{e}}$ siècle $\left(?^{23}\right)$ & Gell., 4, 5, 1-6 \\
\hline 398 & Liv., 5, 15 ; Dion. Hal., Ant., 12, 11-12 ; Plut., Cam., 4 ; Cic., Div., 1, 100 \\
\hline 362 & Varro, Ling., 5, 148 \\
\hline 296 & Cass. Dio, 8 in Zon., 8,1 \\
\hline $279 / 273$ & Liv., Per., 14 ; Cic., Div., 1, 16 \\
\hline 214 & Liv., 24, 10, 6-13 \\
\hline 207 & Liv., 27, 37, 5-10 \\
\hline
\end{tabular}




\begin{tabular}{|c|c|}
\hline 199 & Liv., $32,1,10-14$ \\
\hline 192 & Liv., 35, 21, 2-5 \\
\hline 191 & Liv., $36,37,2$ \\
\hline 186 & Obseq., 3 \\
\hline 182 & Liv., $40,2,1-4$ \\
\hline 177 & Liv., $41,13,1-3$ \\
\hline 172 & Liv., $42,20,1-6$ \\
\hline 171 & Plin., Nat., 7, 36, repris dans Gell., 9, 4, 13-15 \\
\hline 152 & Obseq., 18 \\
\hline 142 & Obseq., 22 ; Oros., $5,4,8$ \\
\hline 137 & Oros., $5,4,19$ \\
\hline 136 & Obseq., 25 \\
\hline $\begin{array}{l}130 \text { (ou plutôt } \\
\left.129^{24}\right)\end{array}$ & 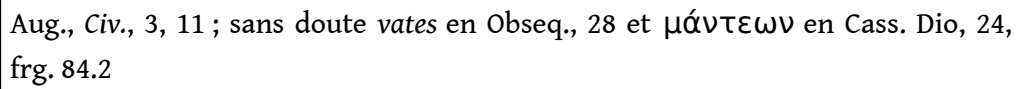 \\
\hline 126 & Obseq., 29 \\
\hline 117 & Cic., Div., 1, 97-98 \\
\hline 104 & Obseq., 43 \\
\hline 102 & Obseq., 44 \\
\hline 99 & Obseq., 46 \\
\hline $92\left(?^{25}\right)$ & Diod., 32, 12, 2 (Photius, Bibl., p. 379a Bekker) \\
\hline $91-90$ & Cic., Div., 1, 99 et 2, 59 ; cf. Plin., Nat., 2, 199 \\
\hline 88 & Diod., 38/39, frg. 5 in Ioann. Antioch. 17-18 Lambros \\
\hline 83 & Obseq., 57 \\
\hline 65 & 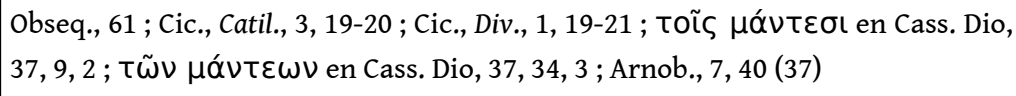 \\
\hline 56 & 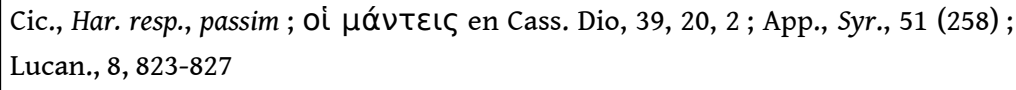 \\
\hline Entre 56 et 52 & Phlegon., Olym., frg. 13 \\
\hline
\end{tabular}




\begin{tabular}{|c|c|}
\hline 54 & Plin., Nat., 2, 147 \\
\hline 43 & App., Civ., 4, 1, 4 (14-15) \\
\hline $\begin{array}{l}\text { Sous } \quad \text { Tullus } \\
\text { Hostilius }\end{array}$ & $\begin{array}{l}\text { La première pluie de pierres connue à Rome fut conjurée, rapporte Tite-Live, soit } \\
\text { d'après une voix céleste, soit sur l'avis des haruspices (seu voce caelesti ex Albano } \\
\text { monte missa... seu haruspicum monitu, Liv., 1,31, 4). }\end{array}$ \\
\hline 483 & 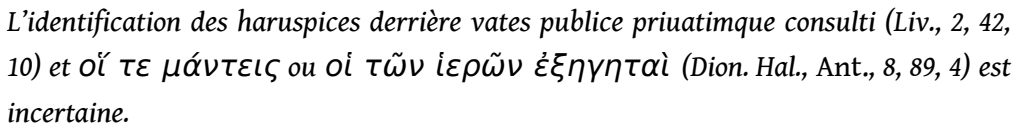 \\
\hline 472 & 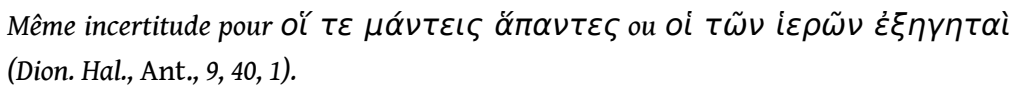 \\
\hline 122 & 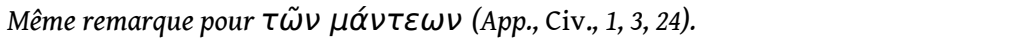 \\
\hline 114 & 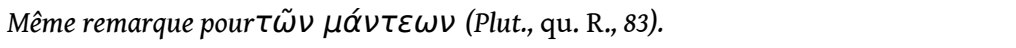 \\
\hline 91 & 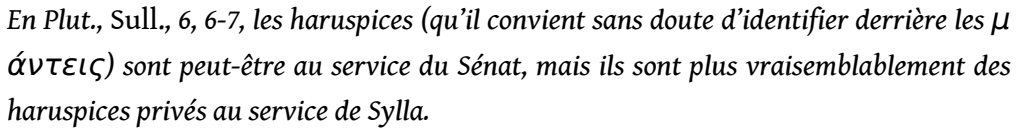 \\
\hline 84 & 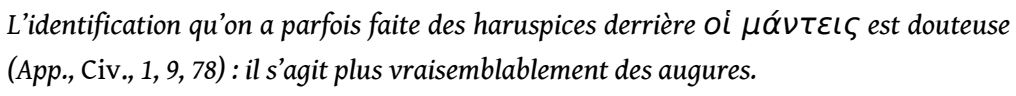 \\
\hline 48 & 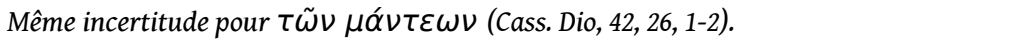 \\
\hline 47 & 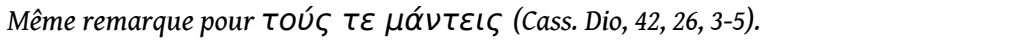 \\
\hline
\end{tabular}

\section{BIBLIOGRAPHIE}

BLOCH R. (1949), « Les prodiges romains et la procuratio prodigiorum », RIDA 2, p. 119-131 (Mélanges Fernand de Visscher 1).

BLOCH R. (1963), Les prodiges dans l'antiquité classique, Paris.

BLOCH R. (1991), La divination. Essai sur l'avenir et son imaginaire, Paris.

BOUCHÉ-LECLERCQ A. (1871), Les pontifes de l'ancienne Rome, Paris.

BOUCHÉ-LECLERCQ A. (1882), Histoire de la divination dans l'Antiquité, Paris, t. 4.

CHAMPEAUX J. (1996), « Pontifes, haruspices et décemvirs : l'expiation des prodiges de 207 », REL

74, p. 67-91. 
ENGELS D. (2007), Das römische Vorzeichenwesen (753-27 v. Chr.). Quellen, Terminologie, Kommentar, historische Entwicklung, Stuttgart (PAwB 22).

FLOBERT A. (1995-1999), Tite-Live. Histoire romaine, Paris, 7 vol.

HAACK M.-L. (2003), Les haruspices dans le monde romain, Bordeaux (Ausonius-Scripta Antiqua 6).

HANO M. (1986), « Haruspices et vates chez Tite-Live », Caesarodunum Suppl. 56, p. 101-121.

LEHMANN Y. (1999), « Divination et prédestination à Rome. Enjeux doctrinaux et politiques », dans Smadja E. \& Geny E. éd., Pouvoir, divination et prédestination dans le monde antique. Tables rondes internationales de Besançon, février 1997/mai 1998, Besançon, p. 249-258.

LINDERSKI J. (1995), Roman Questions. Selected Papers, Stuttgart (HABES 20).

MACBAIN B. (1982), Prodigy and expiation : a study in religion and politics in Republican Rome, Bruxelles (coll. Latomus 177).

ROSENBERGER V. (1998), Gezähmte Götter. Das Prodigienwesen der römischen Republik, Stuttgart (HABES 27).

SCHEID J. (1994), « Le temps de la cité et l'histoire des prêtres, des origines religieuses de l'histoire romaine », dans Detienne M. dir., Transcrire les mythologies, Paris, p. 149-158.

SCHEID J. (1998), La religion des Romains, Paris.

SCHEID J. (1999), « Hiérarchie et structure dans le polythéisme romain. Façons romaines de penser l'action », ARG 1-2, p. 184-203.

SCHEID J. (2001), Religion et Piété à Rome, Paris.

VAN HAEPEREN F. (2002), Le Collège pontifical ( $3^{e}$ s. a. C. - $4^{e}$ s. p. C.). Contribution à l'étude de la religion publique romaine, Bruxelles-Rome.

VIGOURT A. (2001), Les présages impériaux d'Auguste à Domitien, Paris.

WÜLKER L. (1903), Die geschichtliche Entwicklung des Prodigienwesens bei den Römern. Studien zur Geschichte und Überlieferung der Staatsprodigien, Leipzig.

\section{NOTES}

1. Type de signe oblatif exprimé, en latin, non seulement par prodigium, mais aussi par portentum, ostentum, monstrum, etc. (BLOCH 1991, p. 76 sq.).

2. BLOCH 1949, p. 120 sq.; BLOCH 1991, p. 75. La frontière entre "prodige » et "présage » (ce dernier étant généralement désigné par le terme omen : ibidem, p. 68-70) était cependant parfois délicate à définir pour les Romains eux-mêmes. Tite-Live qualifie ainsi le signe constitué par l'enseigne romaine inarrachable avant Trasimène à la fois d'omen et de prodigium (Tite-Live, 22, 3, 11 et 14). Voir BOUCHÉ-LECLERCQ 1882, p. 75-77 et ENGELS 2007, p. 43-47 et 279-282. Complique encore la distinction entre "prodige» et "présage» la tendance des haruspices sollicités par les autorités romaines à interpréter les prodiges comme des signes, favorables ou défavorables, de l'avenir (MACBAIN 1982, p. 125 sq.). C'est en revanche très rarement le cas des prêtres romains qui interprètent généralement le prodige comme l'avertissement d'une rupture de la pax deorum. La dimension oraculaire des Livres Sibyllins amène certes parfois les (quin)décemvirs - mais cela est rare - à interpréter les prodiges comme des présages : Denys d'Halicarnasse, Antiquités romaines, 10, 2, 2-6 (461 av. J.-C.) ; Plutarque, Questions romaines, 83 (114 av. J.-C.) ; Pline l'Ancien, Histoire Naturelle, 17, 243 (50 av. J.-C.) ; Cicéron, De la divination, 1, 97-98 (en général) ; et peut-être Dion 
Cassius, 12 in Zonaras, 8, 19 et Tzetzes, ad Lykophr. Alex., 603 (228 av. J.-C.). Il ne faut pas se laisser abuser par les nombreuses interventions d'haruspices après la Seconde Guerre Punique, qui ont pu donner l'impression que les Romains considéraient de plus en plus les prodiges comme des présages (BLOCH 1991, p. 75 ; LEHMANN 1999, p. 255). Leurs interventions nombreuses, qui sont d'abord le reflet d'une meilleure intégration des spécialistes étrusques, dont la formation a été officiellement encouragée par le Sénat au cours de la deuxième moitié du III siècle av. J.-C. et dont une partie semble résider à Rome à partir du II siècle (HAACK 2003, p. 40-44), ne supplantent en effet nullement les interventions des pontifes et des (quin)décemvirs romains, lesquels continuent à interpréter et à expier les prodiges comme auparavant. La perte de Tite-Live pour la période postérieure à 167 av. J.-C., compensée de manière insatisfaisante par Obsequens, qui ne rapporte pas systématiquement l'énoncé des expiations, explique aussi cette fausse impression d'une confusion progressive, à Rome, entre prodiges et présages. Il est juste de dire, en revanche, qu'avec les grands imperatores apparaît une tendance à la monopolisation et à la personnalisation des signes, interprétés de plus en plus souvent, notamment par les haruspices privés qui se mettent à leur service (à distinguer des haruspices publics au service du Sénat: HAACK 2003, p. 51-75), comme des présages de l'avenir de ces ambitieux (ROSENBERGER 1998, p. 214 ; ENGELS 2007, p. 786-797).

3. Pour plus de détails, voir ENGELS 2007, p. 38-59 et 259-282.

4. Tite-Live, 1, 20, 7 : ut idem pontifex edoceret, quaeque prodigia fulminibus aliove quo visu missa susciperentur atque curarentur («Le pontife devait aussi donner des éclaircissements (...) sur la signification des prodiges produits par la foudre ou par quelque autre phénomène et sur le moyen de les conjurer »). Les traductions sont empruntées à Annette FLOBERT (1995-1999).

5. Cf. annexes.

6. Tite-Live, $25,7,9$ : «Il y eut une journée de prières publiques pour conjurer ces prodiges ».

7. Tite-Live, 27, 11, $6:$ « Ces prodiges furent conjurés par le sacrifice de victimes adultes ».

8. Tite-Live, 1, 31, 4: «En tout cas l'habitude se conserva de célébrer les dieux pendant neuf jours, chaque fois que ce même prodige était signalé ».

9. Cf. annexes. S'il reste prudent quant à la représentativité des sources, Veit ROSENBERGER (1998, p. 54) a souligné encore récemment, sans en mesurer tout le paradoxe, la rareté des interventions pontificales : «bis zum Ende des zweiten Jahrhunderts v.Chr. vorwiegend die Decemviri, danach eher der Haruspices konsultiert wurden; die Pontifices sind generell selten belegt. Doch sollte dieser Sachverhalt aufgrund der disparaten Überlieferungslage nicht überbewertet werden ».

10. SCHEID 1998, p. 112 sq. ; SCHEID 2001, p. 61 sq. ; VAN HAEPEREN 2002, passim.

11. Tite-Live, $1,20,7$. Cf. n. 4.

12. BOUCHÉ-LECLERCQ 1871, p. 184 sq. ; BLOCH 1963, p. 123 ; CHAMPEAUX 1996, p. 71 sq. Françoise vaN HAEPEREN (2002, p. 240-244) développe une argumentation similaire, fondée sur la brevitas de TiteLive, lorsqu'elle suggère une intervention pontificale dans les cas d'instauratio de cérémonies religieuses publiques.

13. Cf. n. 2.

14. Voir, par exemple, Tite-Live, $22,57,4$ : hoc nefas cum inter tot, ut fit, clades in prodigium versum esset, "cette faute, au milieu de tant de malheurs, passa pour un prodige, comme il arrive souvent "; Tite-Live, 40, 2, 3: itaque in prodigium versa ea tempestas procurarique haruspices iusserunt, «cet orage fut considéré comme un avertissement des dieux [prodigium] et les haruspices ordonnèrent des mesures expiatoires ".

15. Voir Tite-Live, 1, 31, 4. Cf. n. 8.

16. La modification apportée à la traduction d'Annette Flobert est indiquée entre crochets : j'ai préféré traduire urbs lustrata par « on procéda à la lustration de la ville » que par «la ville fut purifiée », car la lustration n'est pas une purification (sCHEID 1999, p. 187).

17. Voir Tite-Live, 22, 1, 15-16 ; 37, 3, 1-5 et 40, 19, 4. 
18. Toutes les dates se situent avant notre ère.

19. Les interventions incertaines sont indiquées, en italique, à la fin de chacune des trois listes. Le caractère historique des interventions les plus anciennes est évidemment sujet à caution. Sauf exceptions, les abréviations sont reprises à la Neue Pauly.

20. ENGELS 2007, p. 352 et n. 54.

21. Ibid., p. 355.

22. Seules ont été prises en compte les interventions publiques des haruspices, sur convocation du Sénat.

23. ENGELS 2007, p. 714.

24. Ibid., p. 547.

25. Ibid., p. 579 et n. 234.

\section{RÉSUMÉS}

Bien que les sources évoquent explicitement le rôle primordial des pontifes dans la prise en charge des prodiges, les piacula pontificaux sont rarement attestés, contrairement à ceux des (quin) décemvirs sacris faciundis et des haruspices. Il convient de résoudre ce paradoxe en attribuant les nombreuses procurationes anonymes non pas au Sénat, comme cela a souvent été fait, mais aux pontifes. Un faisceau d'indices abonde dans ce sens: la nécessité du consilium sacerdotal dans toute affaire religieuse; les indices qui permettent de supposer que la chronique annuelle $\mathrm{du}$ collège pontifical, où étaient enregistrés notamment les prodiges, revêtait la forme d'un décret sacerdotal; les traces d'une consultation systématique des pontifes en amont de la consultation éventuelle des (quin) décemvirs et des haruspices pour des expiations complémentaires; enfin, l'existence d'une grande similitude entre les listes de prodiges à conjuration anonyme et les listes à conjuration pontificale.

Although the sources explicitly evoke the primordial role of the pontiffs in the care of prodigies, the pontifical piacula are seldom attested, contrary to those of the (quin) decemviri sacris faciundis and the haruspices. This paradox should be solved by assigning the many unspecified procurationes not to the Senate, as it was often done, but to the pontiffs. Several clues allow such a hypothesis : the necessity of a sacerdotal consilium in any religious affair ; the clues which let us assume that the annual chronicle of the pontifical college, which recorded in particular the prodigies, took the form of a sacerdotal decree ; traces of a systematic consultation of the pontiffs before the possible consultation of the (quin) decemviri and the haruspices for complementary expiations; eventually, the existence of a great similarity between the lists of prodigies anonymously cared and the lists cared by the pontiffs.

\section{INDEX}

Mots-clés : divination, prodige, pontife, procuratio, piacula

Keywords : divination, prodigy, pontiff, procuratio, piacula 
AUTEUR

YANN BERTHELET

Université Paris 1 Panthéon-Sorbonne, ANHIMA - UMR 8210 